\title{
Rat sciatic nerve axoplasm proteome is enriched with ribosomal proteins during regeneration processes
}

Andres Di Paoloł‡, Joaquina Farias $\$$ § Joaquin Garat ${ }^{\ddagger}$, Andrew Macklin", Vladimir Ignatchenko", Thomas Kislingerll, $\perp^{*}$, José Sotelo Silveira ${ }^{\ddagger}, \# *$

† Departamento de Proteínas y Ácidos Nucleicos, IIBCE, 11600, Montevideo Uruguay.

‡ Departamento de Genómica, IIBCE, 11600, Montevideo Uruguay.

§ PDU “Espacio de Biología Vegetal del Noreste", Centro Universitario de Tacuarembó, UdelaR, Tacuarembó, Uruguay (present address)

|| Princess Margaret Cancer Research Tower, 101 College Street, Room 9-807 Toronto, ON M5G 1L7, Canada

$\perp$ University of Toronto, Department of Medical Biophysics, University of Toronto, 101 College Street, Toronto, ON M5G 1L7, Canadá

\# Departamento de Biología Celular y Molecular, Facultad de Ciencias, 11400, Montevideo Uruguay 
* Corresponding authors: José Sotelo Silveira. Address: Avenida Italia 3318, 11600. email: jsotelosilveira@iibce.edu.uy or sotelojos@gmail.com Phone number: +59824871616.

Thomas Kislinger. Address: 101 College Street, Room 9-807 Toronto, ON M5G 1L7, Canada. e mail: thomas.kislinger@utoronto.ca Phone number: 416-581-7627.

\section{SUPPORTING INFORMATION TABLE OF CONTENTS.}
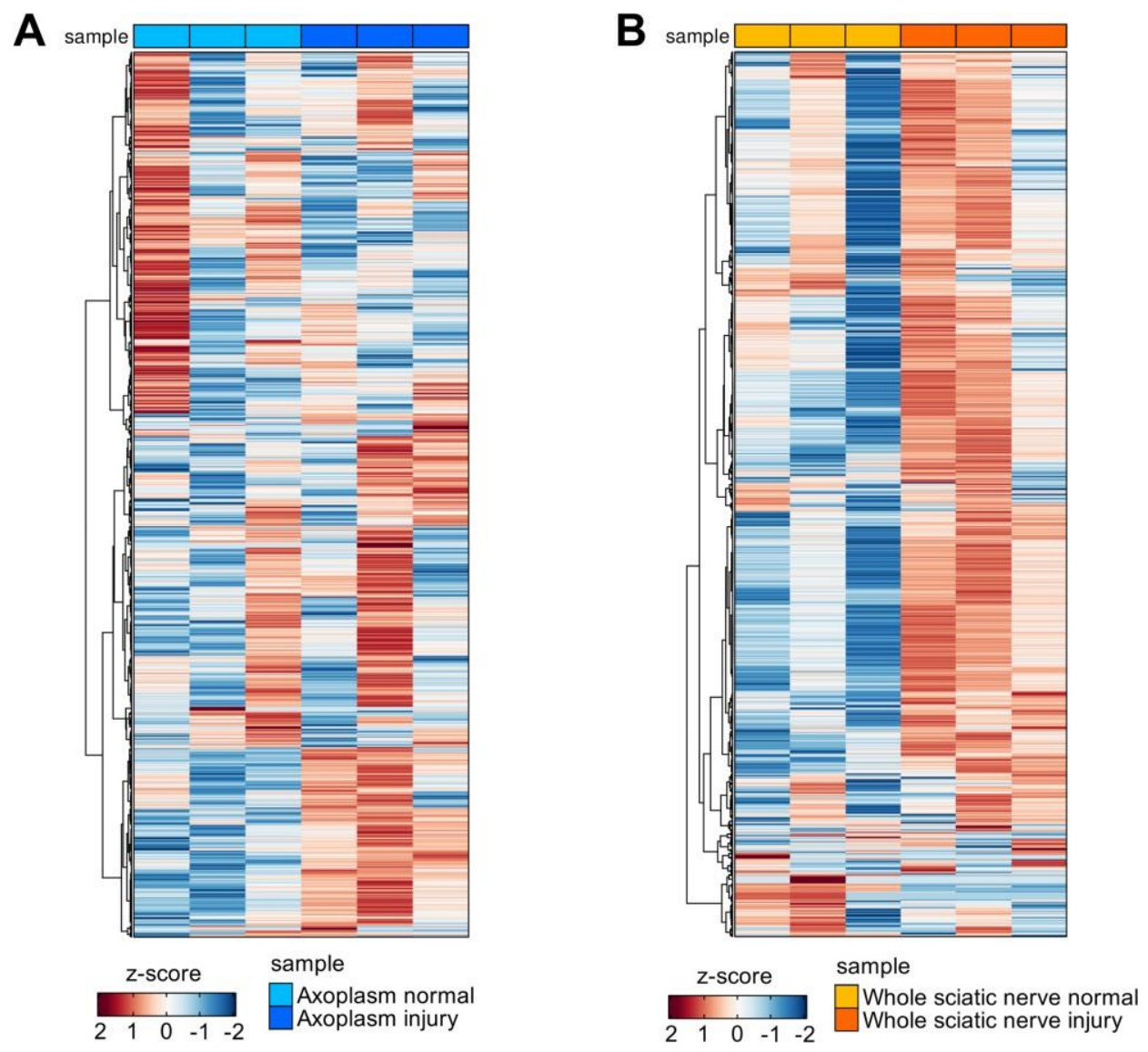

Supplementary Figure 1. Heatmaps of axoplasm replicates (A) and whole nerve sample replicates (B) plotted separately. 


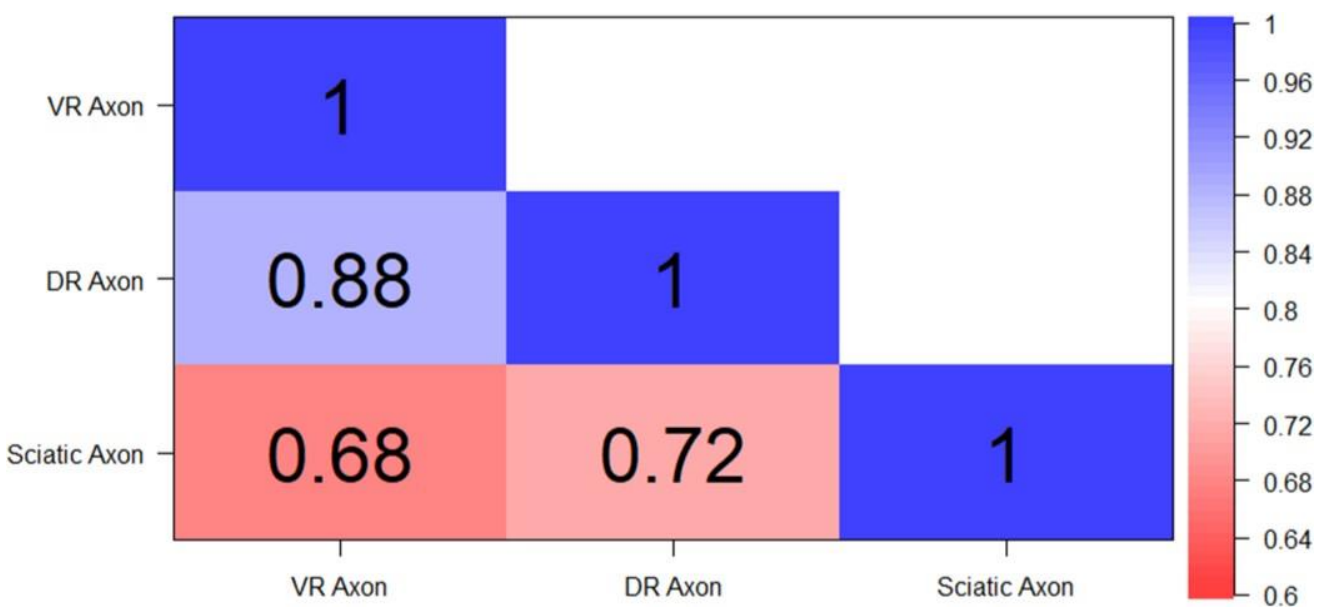

Supplementary Figure 2. Pearson correlation coefficient for axon samples comparison.

A

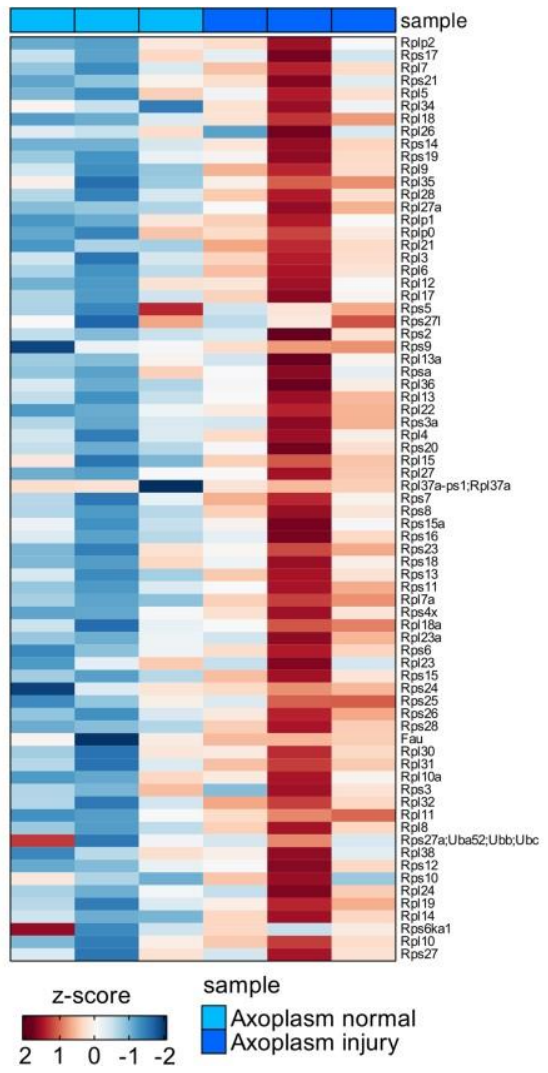

B

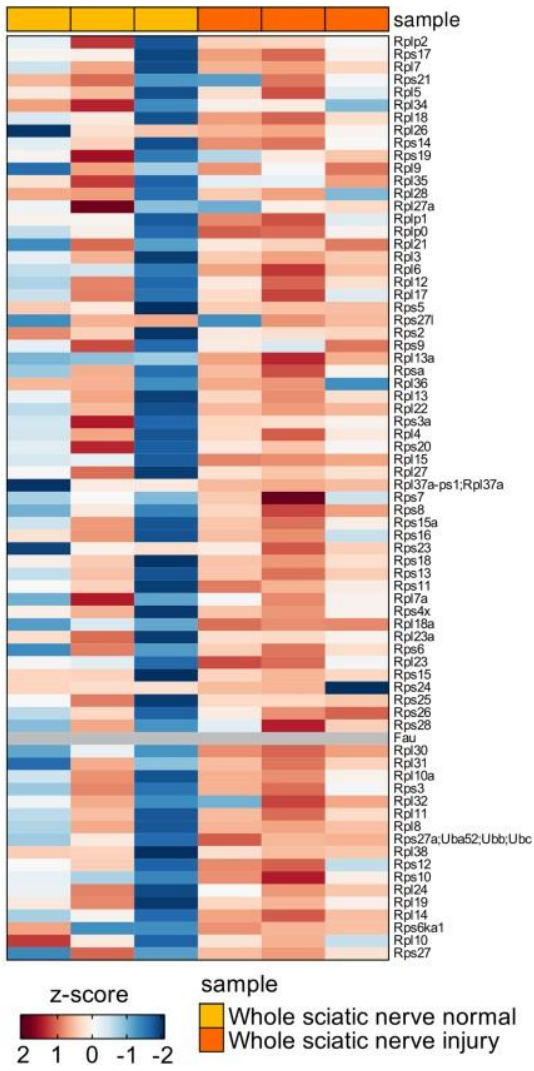

Supplementary Figure 3. (A) Heatmap for the 73 ribosomal proteins detected in the axoplasm samples at normal and injury conditions expressed in iBAQ values. (B) The same as (A) but for whole sciatic nerve samples. 
A Axoplasm Data Hypotonic Extraction (Rishal et al)

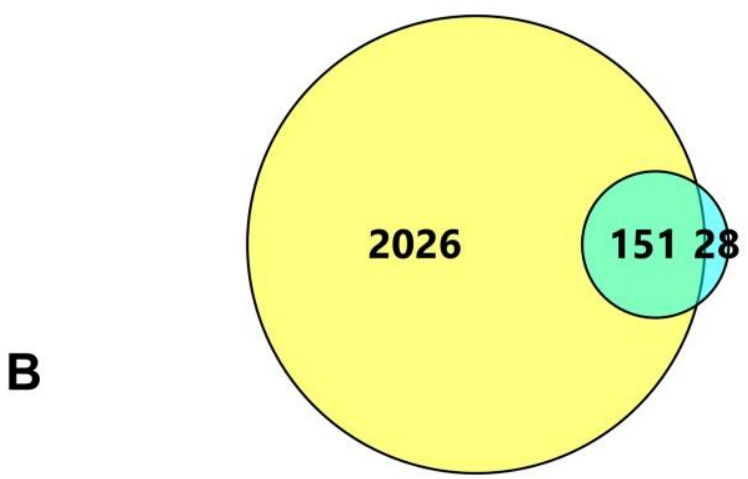

Axoplasm Sample

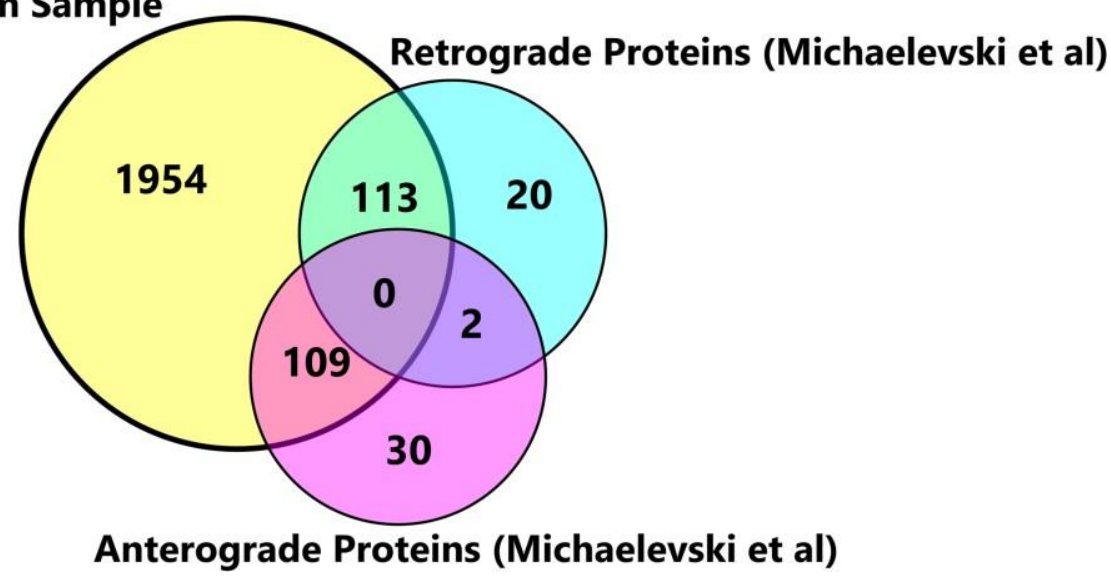

Supplementary Figure 4. Overlap between axoplasm sample and the proteomes detected

by extruded axoplasm extraction in previous articles.

Supplemental Table 1. Sciatic samples raw data and statistical analysis.

Supplemental Table 2. Ventral and dorsal roots raw data and statistical analysis.

Supplemental Table 3. GO Biological Processes for each comparison.

Supplemental Table 4. Heatmap clusters for axon, mixed and whole nerve related protein 
groups.

Supplemental Table 5. List of Protein groups ordered by iBAQ values.

Supplemental Table 6. Differential expressed proteins at ventral root vs dorsal root axoplasm proteomes.

Supplemental Table 7. List of all ribosomal proteins detected in each comparison.

Supplemental Table 8. List of enriched proteins for all different comparisons.

Supplemental Table 9. Protein groups only enriched at injured axons.

Supplemental Table 10. Lists of protein groups in common and only detected in our axoplasm dataset.

Supplemental Table 11. List of RBPs detected in axoplasm samples. 